\title{
Enfermedad periodontal en embarazadas adolescentes. Revisión bibliográfica
}

Antonieta Cotis ${ }^{1}$

María Elena Guerra ${ }^{2}$

\section{Resumen}

Objetivo: Indagar en la literatura información sobre la enfermedad periodontal en adolescentes embarazadas, que le permitan al odontopediatra opininar acerca de los diferentes hallazgos según las patología periodontales más prevalentes en estas pacientes. Materiales y métodos: Se rasteo literatura impresa en libros y revistas, así como búsqueda de artículos publicados en internet, desde 1963 hasta 2013, en español e ingles se tomó en consideración el periodo de embarazó en la adolescencia. Resultados: Se encontraron 40 publicaciones sobre enfermedad periodontal y embarazo, de los cuales solo 4 se referian a adolescentes, los autores coinciden en que se requiere una mayor atención interdisciplinaria, que incluya al Odontólogo, no solo por la salud bucal de la madre gestante sino para asegurarle mejores condiciones de desarrollo del proceso gestacional y el hijo que está en formación, así como existe controversia sobre la influencia del embarazo sobre la enfermedad periodontal. Conclusión: Es bien sabido que los cambios hormonales durante, la adolescencia, el embarazo, producen alteraciones sobre el periodonto, que se verían agravados por condiciones desfavorables de higiene oral. El odontopediatra como profesional de la salud que atiende a los adolescentes, debe cuidar la cavidad bucal de la adolescente y su hijo en formación, lo más importante es educar y prevenir a la paciente y su representante, sobre los estados fisiológicos de la mujer puede tener efectos sistémicos y locales que abarcan la cavidad bucal.

Palabras clave: Embarazo, Adolescentes, Enfermedad Periodontal.

Artigo de revisão

\section{Doença periodontal em gestantes adolescentes. Revisão da literatura}

\section{Resumo}

Objetivo: Procurar na literatura informação sobre doença periodontal em gestantes adolescen- tes, que permitam aos odontopediatras opinar acerca dos diferente achados sobre as patologias periodontais mais prevalentes nestas pacientes. Materiais e métodos: Levando em consideração

\footnotetext{
${ }^{1}$ Especialista en Periodoncia Docente Investigadora Departamento de Estomatología Integral del Adulto. Facultad de Odontología. Universidad Santa María.

${ }^{2}$ Dra. En Ciencias Mención Salud Bucal Materno Infantil. Docente Investigadora. Centro de Atención a Pacientes con Enfermedades Infectocontagiosas. Facultad de Odontología Universidad Central de Venezuela.
} 
o período de gestação na adolescencia, se buscou a literatura impressa em livros e revistas, assim como foram buscados artigos publicados na Internet, de 1963 a 2013, em espanhol ou inglês. Resultados: Foram encontradas 40 publicações sobre alterações periodontais e gestação, das quais somente 4 se referiam a adolescentes. Os autores concordam que se requer uma maior atenção interdisciplinar, incluindo o dentista, não apenas para a saúde bucal da mãe gestante, mas também para asegurar melhores condições de desenvolvimento da processo gestacional e do filho que está em formação. Existe também controvérsia sobre a influência da gestação na doença periodontal. Conclusões: É bem conhecido que as mudanças hormonais durante a, adolescencia e a gestação produzem alterações periodontais, que que seriam agravadas por condições desfavoráveis de higiene oral. $\mathrm{O}$ odontopediatra como profissional da saúde que atende os adolescentes debe cuidar da cavidade bucal da adolescente e do seu filho em formação. O mais importante é educar e prevenir às pacientes e seus responsáveis que os estados fisiológicos da mulher podem ter efeitos sistémicos e locais que envolvem a cavidade bucal.

Palavras chave: gravidez, Adolescentes, Doença Periodontal.

Review article

\section{Periodontal disease in pregnant teenagers. Literature Review}

\begin{abstract}
Objetive: Investigate the literature information on periodontal disease in pregnant teenagers, whom will allow the infection about the different findings according to the most prevalent periodontal pathology in these patients. Materials and methods: Look for printed literature in books and magazines, as well as search for articles published on the internet, it took into consideration the period of pregnancy in adolescence. Results: There were 40 publications on periodontal disease and pregnancy, from 1963 until 2013, in Spanish and English of which only 4 relate to adolescents, the authors agree that there is a need for greater interdisciplinary care, which include the dentist, not only by the oral health of the pregnant mother but to assure best conditions for the development of gestational
\end{abstract}

process and the child who is in training, as well as controversy exists over the influence of pregnancy on the periodontal disease. Conclusion: It is well known that hormonal changes during adolescence, pregnancy, produce alterations on the periodontium, which would be compounded by unfavorable conditions of oral hygiene. The pediatric dentist as a health care professional that caters to teenagers, you must take care of the oral cavity of the adolescent and his son in training, the most important thing is to educate and prevent to the patient and their representative, on physiological states of women can have local and systemic effects that cover the oral cavity.

Key Words: pregnancy, adolescents, Periodontal Disease. 


\section{Introducción}

En relación con la embarazada, los cambios más frecuentes y marcados radican sobre el tejido gingival, dando lugar a la llamada gingivitis del embarazo, también al granuloma del embarazo, que es otra alteración del tejido gingival ante la presencia de irritantes locales, En el segundo mes de gestación comienzan los síntomas y signos inflamatorios, que se mantienen o aumentan durante el segundo trimestre, llegan al máximo al octavo mes, descienden durante el último, $\mathrm{y}$ remiten después del parto ${ }^{1 ; 2 ; 3}$.

La Patogénesis de la Enfermedad Periodontal se debe a la acción nociva de los microorganismos y sus productos, por lo tanto la respuesta inmune inflamatoria del huésped, que en el embarazo puede pasar de una respuesta protectora a una respuesta destructiva ${ }^{4}$.

Recientes estudios han demostrado, que la periodontitis puede producir alteraciones en la salud sistémica, ya que el periodonto puede servir de reservorio para bacterias, productos bacterianos y mediadores inmunoinflamatorios, los cuales pueden interactuar con órganos a distancia de la cavidad bucal como en: Enfermedad Cardiovascular, Partos prematuros, bajo peso al nacer, Enfermedad respiratoria Neumonía aspirativa, Control de la glucosa de pacientes diabéticos ${ }^{2 ; 5}$.

En Venezuela ha aumentado el número de adolescentes embarazadas. Los datos del Ministerio del Poder Popular para la Salud durante los años 2010 y 2011 indican que el 23,06\% de los nacimientos anuales corresponden a niñas-madres con menos de 19 años de edad, en este sentido hay que hacer especial atención a dos condiciones fisiológicas que se unen: la adolescencia y el embarazo, ambas son predisponentes de trastornos gingivales ${ }^{6}$.
Por lo anterior no motivó a realizar uan revisión para correlacionar el embarazo y las enfermedades periodontales.

\section{Antecedentes}

Desde 1963, Lôe y Silness estudiaron la prevalencia y severidad de la enfermedad gingival en mujeres gestantes. Reportaron el aumento de Gingivitis después del $2^{\circ}$ mes de embarazo, incrementándose hasta el 8º . En 1964 continuaron el estudio, estableciendo una correlación entre las hormonas, la higiene oral y la condición periodontal de las gestantes, así como la acción que ejerce la placa bacteriana y señalaron que contribuye indirectamente al desarrollo de la enfermedad periodontal ${ }^{8}$.

Cohen y col. en $1969^{9}$. Señalan que el aspecto clínico periodontal, durante el embarazo se divide en tres períodos. El primero y el último son los más vulnerables a producir patológicas, incluso la posibilidad de interrupción del embarazo. Durante la gestación, la inflamación gingival se incrementa en forma significativa desde el inicio, con un aumento de la profundidad de sondaje, sangramiento al sondaje o provocado por la estimulación mecánica como la masticación y el cepillado, el flujo del fluido crevicular y la movilidad dentaria.

Anders Hugoson en $1970^{10}$ encontró que los niveles hormonales gestacionales, eran factores modificadores de la enfermedad gingival en embarazadas.

Socransky y Manganiello en $1971^{11}$ evidenciaron que las células del epitelio de unión, liberan al medio exterior citoquinas proinflamatorias como la interleuquina(IL) 1, la IL-8 y el Factor de Necrosis Tumoral $\alpha$ (TNF $\alpha$ ) entre otros. Se puede asumir que la respuesta inmune ante di- 
chos microorganismos, se da en todo momento durante la vida aún en el periodonto sano. Estos consideran que la manifestación bucal más frecuente durante el embarazo es la enfermedad periodontal entre un 35 a $100 \%$. La influencia hormonal actúa como una respuesta inflamatoria intensa con un aumento en la permeabilidad vascular y la exudación, que provoca el éxtasis de la microcirculaciòn, lo cual favorece la filtración de líquidos en los tejidos perivasculares.7;12

Entre 1988 a 1994, se realizó en Los Estados Unidos de Norteamerica, la tercera encuesta de Salud y Nutrición, (NHAMES III) a fin de dar estimados del estado de salud dental poblacional; Esta encuesta la utilizó el Instituto Nacional de Investigación Craneofacial (NIDCR) para hemorragia o sangrado gingival, concluyendo que un $54 \%$ de jóvenes desde los 13 años tenían sangrado gingival. Siendo más prevalente en grupos entre 14 a 17 años. Se concuye que los adolescentes presentan mayor prevalencia de gingivitis que los niños pre púberes o adultos. Se sospecha que la elevación de las hormonas sexuales en la adolescencia, es la causa de mayor prevalencia porque afecta la composición de la microflora subgingival ${ }^{13}$.

Nakagawa S y col., en el año 1994, en un estudio de ambos sexos con gingivitis y sin gingivitis en etapa pre puberal y puberal, con el objetivo de evaluar los efectos de las hormonas sobre parámetros clínicos y microbiológicos. Encontraron mayores niveles de testosterona sérica en varones y de estradiol y progesterona en hembras, positivamente correlacionadas con una mayor concentración de patógenos periodontales tales como: Prevotella intermedia y Prevotella nigrenses, y entre los parámetros clínicos de la inflamación gingival. ${ }^{14}$

Bryan Whitaker $1994^{15}$, asocia, el comienzo de la gingivitis del embarazo con el incremento hor- monal y el crecimiento selectivo de patógenos periodontales como Prevotella intermedia, en la placa subgingival a partir del tercer o cuarto mes de embarazo. Las hormonas esteroideas actúan como factores de crecimiento para dichas bacterias anaerobias Gramnegativas. Las embarazadas sufren gingivitis gravídica con una frecuencia de 50 a $100 \%$. Se da en mujeres que con anterioridad no tenían inflamación gingival o bien agrava una inflamación ya existente, con carácter hiperplasico, papilas gingivales congestivas, edematosas, con manifestación de dolores y sangrados provocados o espontáneos.

Debido al amplio espectro con que la Enfermedad Periodontal puede presentarse, se hizo imprescindible acogernos a una clasificación rigurosa de esta entidad clínica, y encontramos que en la clasificación de la American Academy of Periodontology (AAP) del año 199916, de enfermedades y lesiones periodontales que es la actualmente aceptada, se describen dos grandes grupos de patologías con características bien diferenciadas: Gingivitis y Periodontitis partiendo de que entre ambas entidades la resorción ósea, remodelación celular de componentes minerales y de la matriz ósea, diferencia ambos grupos de patologías; esta remodelación ósea en estado de salud esta controlada por varias hormonas entre ellas las esteroideas sexuales.

Gemco y col $2000^{17}$ señaló que en esta clasificación de la AAP, no se toma en cuenta la edad ni la progresión de la enfermedad y se clasifican en los siguientes grupos:

- Gingivitis (asociada o no a la placa dental).

- Periodontitis Crónica (localizada o generalizada).

- Periodontitis agresiva (localizada o generalizada). 
- Enfermedades periodontales necrotizante (gingivitis y periodontitis ulcerativa).

- Abscesos del periodonto.

Las enfermedades gingivales inducidas por placa dental modificadas por factores sistémicos. Entre ellos asociados con el sistema endócrino se mencionan: gingivitis de la pubertad, gingivitis del ciclo menstrual. En relación al embarazo: gingivitis, granuloma piógeno y gingivitis de la diabetes.

La Gingivitis es la respuesta inflamatoria de la encía que se produce por la presencia de bacterias que se adhieren en forma de Biopelícula a la superficie del diente, y puede tratarse de un problema exclusivamente inflamatorio, o modificada por factores sistémicos y locales que pueden exacerbar las características clínicas de la gingivitis ${ }^{18 ; 19}$.

Según Markou. E y col $2009^{18}$ en relación con los mecanismos de acción de las hormonas esteroideas sexuales que aumentan durante el embarazo, demostró que directa e indirectamente ejercen influencia sobre la proliferación celular, incluyendo los queratinocitos y los fibroblastos, diferenciación y crecimiento en los tejidos gingivales. Hay dos teorías para explicar la acción de las hormonas en estas células: a) cambio de la efectivida de la barrera epitelial a la actividad bacteriana de la microflora bucal y b) efecto sobre reparación y mantenimiento del colágeno.

La bacteria anaerobia gramnegativa Prevotella intermedia, ha sido relacionada con la inflamación gingival, aumenta en la placa a partir del tercer mes de gestación y utiliza a la progesterona y el estrógeno presentes en el fluido gingival como fuente de alimentos. Ya que se ha descrito la relación directa entre la higiene bucal buena y un mejor estado gingival en las gestantes, aún cuando haya avanzado el embarazo. 19,20;21
El aumento hormonal gestacional produce una alteración del equilibrio fibrinolítico, manifestado por la gingivitis gravídica. La gingivitis es mayor cuantos más jóvenes son las gestantes. En adolescentes entre 14 y 19 años, embarazadas $^{15 ; 22}$, encontraron mayor grado de índice de placa, coincidentemente con mayor secreción de estrógenos y progesterona; El aumento de los niveles séricos de estas dos hormonas inhibe la función de las células inflamatorias contra la placa bacteriana agravando el cuadro de la gingivitis del embarazo.

La maternidad es un momento importante en el ciclo vital femenino. Las embarazadas constituyen un grupo poblacional más vulnerable física y psicológicamente debido a las grandes perspectivas de cambios. El equipo de salud, debe abordar integralmente al individuo basándose principalmente en medidas educativas y preventivas. $^{23}$

En el fluido Crevicular Gingival (FCG), se encuentran inmersos Los microorganismos presentes en el SP, y sirve de escenario para la respuesta inmunológica ante tales microorganismos el FCG de un periodonto sano, contiene diferentes moléculas y células que participan en la respuesta inmunológica Ej. Inmunoglobulinas (Ig) del tipo Ig G e Ig A, lisozimas, células como polimorfonucleares, y restos celulares provenientes de la descamación del epitelio del surco, hoy en día se acepta que también juegan un rol importante en la respuesta inmune innata del hospedero. ${ }^{24 ; 25}$

Paulo Mascarhenas en $2003^{26}$, demostró, el papel hormonal y su rol en la progresión de la enfermedad periodontal, y podía revertir estos efectos o minimizarlos mejorando el control de placa. 
Guerra y col. $2009^{27}$ Señalaron que en grupo de embarazadas venezolanas VIH/SIDA Reportaron una mayor prevalencia de candidiasis $\mathrm{y}$ gingivitis, por lo que se destaca la necesidad del diagnóstico y tratamiento en las embarazadas VIH/SIDA, ya el diagnóstico precoz, podría evitar el progreso de la infección. Entre las recomendaciones indican incorporar programas preventivos que incluyan el control de la Biopelícula, así como la eliminación de irritantes locales, y diagnostico temprano de la enfermedad periodontal. ${ }^{27}$

Berrios Andrea en el $2011^{28}$ señaló la importancia de la hormona relaxina, por ser la encargada de relajar músculos y articulaciones, favoreciendo la elasticidad de la musculatura y la movilidad de los huesos de la pelvis así como el paso del niño por el canal vaginal, y podría causar movilidad dental facilitando el empaquetamiento de alimentos en los espacios interproximales y originando gingivitis, otro aspecto destacado por esta autora es que el incremento del flujo sanguíneo durante el embarazo podría ser la causas de la inflamación y el dolor, e incluso el sangramiento de las encías aumentando el riesgo a presentar gingivitis.

Armitagge en el 201329, hace referencia a un aumento de la severidad de la gingivitis durante el embarazo y la atribuyen a la mayor secreción hormonal, que afecta la micro circulación gingival produciendo cambios en la permeabilidad vascular, resultando en un edema gingival $\mathrm{y}$ respuesta inflamatoria aumentada a la presencia de la placa bacteriana, sobre la cual también actúa, ya que este aumento de secreción hormonal modifica la microbiota bucal aumentando la Prevotella intermedia.

Las enfermedades bucales específicas en las embarazadas establecen la necesidad de adoptar protocolos de tratamiento odontológico durante el embarazo, que incluyan programas preventivos, control de la Biopelicula y eliminación de irritantes locales, se destaca que odontólogo que esté en contacto con embarazadas debe estar capacitado en el manejo de la enfermedad periodontal inicial y en la interconsulta oportuna con el periodoncista, cuando se presenten casos de periodontitis moderada o avanzada. ${ }^{19 ; 20 ; 30 ; 31}$

Según Matesanz-Pérez P y col. $2008^{20}$ en una revisión de la literatura señalan, que la gestación predispone a la mujer a un aumento del apetito y al deseo de alimentos poco habituales. En el primer trimestre de embarazo son frecuentes las nauseas y los vómitos debido a la secreción de gonadotropina coriónica humana y el aumento de estrógenos. Además de los cambios gingivales debidos al aumento de la respuesta inflamatoria durante la gestación, el 0,5 - 9,5\% de las embarazadas experimentan un crecimiento gingival localizado, el granuloma piógeno o "tumor del embarazo". La encía se ve afectada en el $70 \%$, esta lesión no es maligna. En su patogenia se sospecha la acción de los irritantes locales sobre un metabolismo tisular alterado por efecto hormonal. Crecen con rapidez, aunque rara vez alcanzan más de $2 \mathrm{~cm}$ de tamaño, sangran fácilmente y pueden variar del color rojo púrpura al azul oscuro. Tras el parto, las lesiones pueden disminuir o desaparecer. ${ }^{21-22}$

Si bien las bacterias bucales y sus productos son las iniciadoras de la enfermedad periodontal, las citoquinas juegan un papel importante en la patogénesis de enfermedades infecciosas. La enfermedad periodontal se caracteriza por ser un proceso inflamatorio destructivo que afecta las estructuras de soporte del diente. Las citoquinas proinflamatorias como la interleucina 1 (IL-1), el factor de necrosis tumoral alfa (TNFalfa) y el interferón gamma (IFN gamma) son 
considerados los principales mediadores de inflamación crónica, incluída la periodontitis. ${ }^{27}$ Las interleucinas IL-6, IL-8, la prostaglandina E2 y las metaloproteinasas provenientes del infiltrado inflamatorio crónico, se han propuesto como marcadores pronósticos de las patologías periodontales ya que los cambios encontrados en la concentración de las mismas se relacionan con la activación de las células que componen el infiltrado inflamatorio y comprometen la participación del sistema inmune local. En cambio el aumento de citoquinas y moléculas con acción antiinflamatoria como son la IL-4, IL- 10, TGF alfa (factor transformador de crecimiento alfa), IL-1Ra (interleucina-1 receptor antagonista) y TIMP (inhibidor tisular de las metaloproteinasas) garantizan un periodonto sano..$^{33,34}$

Durante el embarazo profundas perturbaciones en la inmunidad innata y adquirida impactan el curso clínico de un número de enfermedades infecciosas, se ha sugerido que las infecciones periodontales pueden aumentar el riesgo de resultados adversos del embarazo. Encontramos en la revisiòn asociasión entre la relación bidireccional embarazo y enfermedad periodontal, así como los posibles mecanismos detrás de esta interacción. ${ }^{35}$

Díaz y Castellano en el 2004 en un grupo de 7.952 mujeres en México reportaron que el embarazo no mostró ser factor decisivo para el desarrollo de enfermedad periodontal, no encontraron diferencias en la prevalencia de Gingivitis (54.54 y $50.50 \%$ ) y Periodontitis (31.82 y $31.75 \%$ ) entre los dos grupos, sin embargo la severidad de Periodontitis fue significativamente mayor en las embarazadas: 18.18 y $9.88 \%(\mathrm{P}<0.01){ }^{34}$

Steven Offenbacher, realizó en $2011^{36}$ estudios aleatorios de ensayos clínicos y no pudo encontrar que la terapia periodontal estándar durante el embarazo reduce la incidencia de los resultados adversos del embarazo (por ejemplo, parto prematuro y bajo peso al nacer). Sin embargo, tratar la enfermedad periodontal durante el embarazo puede ser demasiado tarde para reducir la inflamación gingival que se relaciona con los resultados adversos del embarazo, el tratamiento periodontal puede causar bacteriemia, que podría iniciar resultados adversos del embarazo. Se necesitan ensayos aleatorios futuros para probar si el tratamiento de la enfermedad periodontal durante el embarazo reduce la tasa de partos prematuros y niños de bajo peso al nacer.

\section{Materiales y métodos}

Se realizó una revisión narrativa de la literatura buscando información sobre el manejo odontológico de la embarazada, para analizar lo que ha sido ya estudiado, de manera que nos pudiera servir de base para futuros estudios. Está revisón incluyo libros de texto, revistas indexadas impresas y publicaciones electrónicas, se encontaron publicaciones sobre el tema desde el año 1963 al 2013 en los idiomas ingles y español.

\section{Resultados}

Se encontraron cuarenta (40) publicaciones que habían estudiado el tema, los autores coinciden en que se requiere una mayor atención interdisciplinaria durante el embarazo, nosotros posterior a esta revisión consideramos que se debe incluir al Odontopediatra, no solo por ser responsable de la salud bucal de la adolecente gestante, sino para asegurarle mejores condiciones de desarrollo de la madre y el niño en formación, ya que es conocido que aproximadamente alrededor de la sexta semana de vida empieza la formación dental. 
Durante está revisión solo se encontraron cuatro (4) publicaciones que relacione la enfermedad periodontal durante embarazo en la adolescencia.

Una realizada en 2001, por Casnati, Ana María y cols. Quienes señalan que El 77\% de las adolescentes presentó una higiene bucal insuficiente y el 65\% no recibió atención, 65\% presentó enfermedad periodontal incipiente, 7,3\% enfermedad periodontal franca en las pacientes de 17 y 18 años, algunas embarazadas por segunda vez. ${ }^{37}$

La segunda del año 2005, por Guzmán Vera Yanina con el objetivo de determinar la relación que existe entre la severidad de la inflamación gingival de las adolescentes y adultas embarazadas. Los resultados mostraron que la severidad de la inflamación gingival fue mayor en adolescentes que en adultas. El mayor promedio de los índices gingival y de placa se encontró en adolescentes con diferencias estadísticamente significativas $^{4}$.

La tercera fue un estudio realizado Herrera C y Alejandro D. en 2011, sobre la prevalencia de enfermedad periodontal en adolescentes de 15 a 18 años de edad en la ciudad de Quito. Se concluyó que $98.5 \%$ de la población examinada presentó algún grado de enfermedad periodontal, 100\% necesitan instrucción de higiene bucal y profilaxis, $18.5 \%$ requieren de una remoción de cálculos supra y subgingival y $4.5 \%$ necesitan raspado y alisado radicular. ${ }^{38}$
La cuarta en 2012 BaudoJ y cols. Concluyen que el aumento de los niveles séricos de estas dos hormonas inhiben la función de las células inflamatorias contra la placa bacteriana agravando el cuadro de la gingivitis del embarazo. ${ }^{39}$

Algunos autores enfatizan la educación sexual y acceso a la anticoncepción a los adolescentes. Educar a los proveedores de salud sobre la seguridad y eficacia de la anticoncepción reversible para reducir lembarazos en este grupo etario no deseados ${ }^{40}$.

En la revisión se encontraron datos, limitados sobre el efecto potencial del cuidado en relación a higiene bucal y tratamiento periodontal durante el embarazo, así como también sobre la salud periodontal, fluido crevicular gingival (GCF) y citoquinas séricas y su impacto en los resultados del embarazo.

\section{Conclusión}

Se sabe que los cambios hormonales durante, la adolescencia, el embarazo, producen alteraciones sobre el periodonto, que se verían agravados por condiciones desfavorables de higiene oral. El odontopediatra como profesional de la salud que atiende a los adolescentes, debe cuidar la cavidad bucal de la adolescente y su hijo en formación, lo más importante es educar y prevenir a la paciente y su representante, sobre los estados fisiológicos de la mujer puede tener efectos sistémicos y locales que abarcan la cavidad bucal.

\section{Referencias}

1. Laine M. Effect of pregnancy on periodontal and dental health. Acta Odontol Scand 2002;60(5):257-64.

2. Chicco G, Gennai A, Ceccherini M, Marelli R, Pallassini A. Gingival Changes in pregnancy. Stomatol Mediterr 1989; 9(4):311

3. Banoczy J, Orosz M, Gabris K, Nyarasdy I, Rigo O, Schuder L. Investigation on the correlation of pregnancy, caries and gingivitis. Zahn Mund Kieferheilkd Zentralbl 1978; 66(6):573-81. 
4. Guzmán V Yanina, Severidad de la Gingivitis Asociada al embarazo en adolescente: Tesis Universidad Cayetano Heredia Perú 2005

5. Rotemberg,WilfEnrique,SmaisikFrydman,Karina“Manifestaciones Periodontales de los Estados FIsiologicos de la Mujer". Odontoestomatología 2009 vol.11: 13; Montevideo.

6. Noticias relacionadas con: Venezuela entre los países con más alto índice de embarazo precoz, 2013 www.ultimasnoticias.com.ve consultada 24/08/2014

7. Lôe H, Sillness J; Periodontal Disease in Pregnancy I Prevalence and Severity. Acta Odont Scand: 1963; 21:533-51

8. Sillness J; Lôe H, Periodontal Desease in Pregnancy II. Correlation Between Oral Hygiene and Periodontal Condition Acta Odont Scand: 1964, Vol. 22, (1) 121-135

9. Cohen W; Friedman L; Shapiro J; Kyle GC. A longitudinal Investigation of Periodontal Changes During Pregnacy. J Periodontol 1969; 40: 563-70

10. Hugoson Anders."Gingival Inflamation and Female Sex Hormones." Journal Periodontal Research. 1970;(5):6-18.

11. Socransky, S.S., Manganiello, S.D. The oral microbiota of man frombirth to senility. J Periodontol. 1971; 42(8): 485-96

12. NakawagaS; Fuji H; Machida Y, A Longitudinal Study from prepuberty and puberty of gingivitis: correlation between the occurrence of Prevotellaintermedia and sex hormones. J. Clin. Periodontol 1994; 21:658

13. Enfermedad de las encías o enfermedad periodontal: Causas, síntomas y tratamientos NIHP ubicación No.13-1142S agosto 2013 http: / / www.nidcr.nih.gov Consultada 24/08/1014

14. Carranza. Periodontologia Clínica. Cap. 8.pp116-117. X Edition. Argentina Médica Panamericana 1996

15. Whitaker Bryan. Bouquot J. Alimario A. Identification and semi quantification of estrogen and progesterone receptors in pyogenic granulomas of pregnancy Oral Surgery, Oral Medicine, Oral Pathology 1994; (78) Issue 6; 755-760

16. American Academy of Periodontology 1999 Workshop for a Classification of Periodontal Disease and Conditions. Ann Periodontol 1999; 4: 1- 112

17. Gemco Robert, Goldman y Cohen. . Patogénesis y Respuestas del Huésped en la Enfermedad Periodontal. 2000; Cap.14.

18. Markou E, Eleana B, Lazaros T, Antonios K. The influence of sex steroid hormones on gingiva of women. Open Dent J, 2009; 3: 114-119.

19. Matesanz-Pérez P., Matos-Cruz R., Bascones-Martínez A Enfermedades Gingivales. Una revisión de la Literatura. Avances en Periodoncia 2008; (20) 1 Madrid

20. Díaz Guzmán, Laura María ; Castellanos Suárez, José Luis. Lesiones de la mucosa bucal y comportamiento de la enfermedad periodontal en embarazadas. Medicina oral, patología oral y cirugía bucal, 2004,(9) 5: 430-437

21. Pérez Olga y col. Granuloma piogénico del embarazo. Presentación de un caso.Arch.Argent.Dermatol.2010; 60:155-157

22. Judith Baudo, Sonia Tosti, Patricia Allegretti Niveles séricos de estrógenos y progesterona en relación a la enfermedad gingival en gestantes adolescentes Intra Med Journal,2012; (1) 3

23. Nogueira Coutinho N, Salles Rocha E, Conceicao Ferreira R, Lacerda Vilaca E, Nogueira Moreira A, Silami de Magalhaes C, Control de la enfermedad periodontal y caries en gestantes; Fundación Juan José Carraro, Año 10, № 21, Septiembre / Octubre de 2005, 33-39

24. Figuero- E Ruiz; Prieto-Prieto I; Bascones Martinez A. Cambios Hormonales asociados al embarazo. Afectación Gingvo-periodontal Av Periodont Implantol 2006; 18:101-113

25. Castrillon Laura E. Macin Susana, Palma A. Participación de la Interleucina 1B(1L-1B) en Periodontitis.Revista Odontológica Mexicana.2011(11)4:pp185-200. Diciembre 2011

26. Mascarenhas Paulo y col. Influence of sex hormones on the periodontium. Journal Clinical Periodontology,2003 (30); $8 ; 671-681$

27. Guerra M.E; León JR; Blanco L; Tovar V. Manifestaciones bucales en embarazadas recién diagnosticadas con infección por VIH/SIDA Acta odontol. venez 2009. 47 (3)

28. Berrios Andrea. Manejo Odontológico en pacientes embarazadas agosto 2011. slideshare.net Consultado $12 / 08 / 2014$ 
29. Gary. C Armitagge “Bi-directional relationship between pregnancy and periodontal disease "Periodontology 2000; 61(1): 160-76.

30. Díaz RRM. Modelo de atención estomatológica durante la gravidez (I). Pract Odontol 1986; 8:50-1.

31. Díaz RRM, Martínez SC. Educación para la salud bucal en la madre gestante. Pract Odontol 1989;10:35-7.

32. Pérez Olga y col. Granuloma piogénico del embarazo. Presentación de un caso. Arch. Argent. Dermatol. 2010; 60:155157

33. Castrillon Laura E. Macin Susana, Palma A. Participación de la Interleucina 1B(1L-1B) en Periodontitis. Revista Odontológica Mexicana 2011(11),4:185-200

34. Phillip Vassilopoulus Effect of Intensive Oral Hygiene Regimen During Pregnancy on Periodontal Health, Cytokine Levels, and Pregnancy Outcomes. A Pilot Study. Journal of periodontology 2014; DOI: 10.1902/jop.140248

35. Díaz Guzmán, Laura María ; Castellanos Suárez, José Luis. Lesiones de la mucosa bucal y comportamiento de la enfermedad periodontal en embarazadas. Medicina oral, patología oral y cirugía bucal, 20049 (5): 430-437

36. Steven Offenbacher "Optimal timing of periodontal disease treatment for prevention of adverse pregnancy outcomes: before or during pregnancy?American journal of obstetrics and gynecology 2011; 205(2): 111.

37. Casnati, Ana María; De Ávila, Lilian; Igoa, Alejandra; Lombide, Isabel; Soria, Milka EmbarazoAdolescentey enfermedad periodontal2001https: / / www.academia.edu

38. Coral Herrera, Daniel Alejandro. prevalencia de enfermedad periodontal mediante el registro periodontal simplificado (PSR) en adolescentes de 15 a 18 años de edad del Colegio Nacional Ligdano Chávez de la Ciudad de Quito en el año 2011 https: / / www.dspace.uce.edu Consultada 08/08/2014

39. Judith Baudo, Sonia Tosti, Patricia Allegretti .Niveles séricos de estrógenos y progesterona en relación a la enfermedad gingival en gestantes adolescentes 2012. https: / / journal.intramed. Consultada 08/08/2014

40. Liliana A López-Cano, Sandra L Restrepo-Mesa The relationship-based meaning of teenage pregnancy in Bogotá regarding the family system. Rev Salud Publica (Bogota). 2013 15(6):815-25

Recibido 08/09/2014

Aceptado 18/11/2014

Correspondencia: antonieta.cotis@gmail.com 